\title{
DERIVATIVE UV/VIS SPECTROELECTROCHEMISTRY IN THIN-LAYER \\ REGIME: DECONVOLUTION AND SIMULTANEOUS QUANTIFICATION OF ASCORBIC ACID, DOPAMINE AND URIC ACID.
}

Fabiola Olmo, Jesus Garoz-Ruiz, Alvaro Colina, Aranzazu Heras*

Department of Chemistry, Universidad de Burgos, Plaza Misael Bañuelos s/n, E-09001

Burgos, Spain

ORCID

Jesus Garoz-Ruiz: https://orcid.org/0000-0002-5775-4247

Alvaro Colina: https://orcid.org/0000-0003-0339-356X

Aranzazu Heras: https://orcid.org/0000-0002-5068-2164

Corresponding authors

*E-mail: maheras@ubu.es. Tel: +34 9472588 17. Fax: +34 947258831. 


\section{ABSTRACT}

In this work, UV-Vis spectroelectrochemistry (SEC) in thin-layer regime and in parallel configuration is selected to solve a complex mixture that contains dopamine (DA), ascorbic acid (AA) and uric acid (UA). These molecules, such as many other biological compounds, are acquiring a great importance in analytical and biomedical fields due to the fundamental role that they play in the human metabolism. In addition, low or high levels of these compounds are related with some diseases, highlighting Parkinson's disease. For this reason, the quantification of these biomolecules is becoming increasingly important. However, some drawbacks must be overcome, because the three molecules coexist in the human body and they are interfering species between them. In fact, all of them are oxidized at similar potentials and their UV/Vis absorption bands are overlapped, greatly complicating their quantification. For this reason, derivative SEC together with suitable chemometric tools such as PARAFAC are proposed to solve this complex matrix. This technique allows us to separate the contribution of each of these molecules present in a sample and to quantify all of them, achieving high resolution and reproducibility. Besides, detection limits in the micromolar level are achieved for DA, AA and UA in mixture solutions. To sum up, this work demonstrates the great capability of derivative potentiodynamic SEC combined with the appropriate chemometric tools to solve complex mixtures, a field where SEC is still taking the first steps.

\section{Keywords}

Spectroelectrochemistry, PARAFAC, quantitative analysis, derivative of absorbance with respect to wavelength 


\section{INTRODUCTION}

Dopamine (DA) is a neurotransmitter that participates in the regulation of physiological processes and can be found in the brain. Low levels of DA have been found in patients with Parkinson's disease and high levels in patients with schizophrenia [1, 2]. Meanwhile, ascorbic acid (AA) is an essential vitamin in the human diet that can be found in the brain in presence of different neurotransmitters. Low levels of AA are related, for example, to scurvy $[3,4]$. Lastly, uric acid (UA) is a natural antioxidant. High levels of UA in blood can promote the development of some diseases, such as gout or anemia $[4,5]$. On the other hand, a low level of UA is associated with Parkinson's disease [5]. Undoubtedly, these compounds of biological interest play a fundamental role in human metabolism and their inappropriate amounts are associated with some diseases. For this reason, the study and quantification of these molecules is essential and is becoming increasingly important, particularly in biomedical chemistry and in neurochemistry fields.

Up to now, mainly electrochemical methods have been used to carry out the simultaneous quantification of DA, AA and UA. However, the most important drawbacks of electrochemical methods to determine these compounds are their similar electro-oxidation potentials, the typical presence of the three compounds in the human body and the formation of by-products and side-products during their electrochemical reactions [6]. Thus, due to the high interference that these compounds present between them, the electrode surface is usually modified with different materials that allow shifting the potentials at which these molecules are oxidized. Some of these modifications consist of using, for example, reduced graphene 
oxide (RGO) [7, 8], conductive polymers [9], RGO and conducting polymers nanocomposites [10], nitrogen doped graphene [11], carbon nanotubes (CNTs) and nanoparticles [12], ternary nanocompounds [13] or hexagonal boron nitride [14]. It should be highlighted the increase in selectivity and sensitivity achieved when using modified-electrodes with respect to bare electrodes; however, the modification process of the electrodes is sometimes complex, time-consuming and tedious [6]. In order to overcome these drawbacks, in this work a new method resulting in the combination of electrochemistry and spectroscopy together with the appropriate statistical tools is proposed, using in all experiments pristine electrodes without any type of modification.

Spectroelectrochemistry (SEC) provides simultaneously the electrochemical and spectroscopic information about a system that undergoes an oxidation-reduction process [1, 15-17]. Normal and parallel configurations are the two main optical arrangements for UV-Vis molecular absorption SEC and mainly differ in the direction in which the radiation beam passes through the solution adjacent to the electrode. In the first case, the light beam strikes perpendicularly to the electrode surface, while in the second one it comes parallel to the electrode surface [17-20]. In the present case, parallel configuration is selected due to the several advantages that this arrangement presents with respect to the normal configuration such as the longer optical path, which results in a better sensitivity and lower limits of detection for soluble compounds [15, 21-23]. Taking into account the diffusion regime, SEC in thin-layer regime [24-26] provides the electrochemical and spectroscopic information about the solution changes that occur only in a small confined space adjacent to the working electrode where it is electrolyzed. Consequently, it is much easier to completely 
electrolyze the reactant species. UV/Vis thin-layer SEC is acquiring importance in many research's fields, highlighting its significance in the study of different compounds [24, $26,27]$. It should be emphasized that the importance of SEC for analytical purposes is growing year by year $[1,21,28-31]$, although it is still taking the first steps in this field trying to show its great potential, sometimes with the help of the adequate statistical tools.

In this work, we propose the simultaneous SEC determination of DA, AA and UA in mixtures that include these three molecules, randomly varying their concentrations in order to demonstrate the robustness of the analytical methodology, using bare carbon screen-printed electrodes (SPEs) and without any pre-treatment of the sample or modification of the working electrode surface. In most of the works found in literature, to test the effectiveness of a new electroanalytical method the concentration of two of these compounds is fixed and the other one is determined or the concentration of the three compounds is increased simultaneously. Undoubtedly, this situation is unreal. For this reason, the development of new techniques and/or methodologies should be implemented to allow the determination of these compounds in mixtures with random concentrations in solution. In this way, the determination of real samples can be carried out, where the concentration of these biomolecules can vary significantly depending on the matrix. DA, AA and UA show similarities not only in their oxidation potentials, but also in the position and shape of the absorption spectra of their oxidized forms. Due to the high similarities between their spectra, we propose the derivative UV/Vis absorption SEC with respect to the wavelength as analytical tool to study these mixtures. Derivative spectroscopy is based on plotting the first, second or higher derivative of the absorbance with respect to the wavelength [32]. This 
technique could be the solution to many analytical challenges, such as resolution of complex matrices due to the effective improvement of resolution [33]. Thus, it would allow us to separate compounds with overlapping spectra or to remove the contribution of interfering species [34]. Moreover, the powerfulness of derivative spectroscopy can be extremely increased when coupled with electrochemistry, what is known as derivative SEC. This technique, performing the derivative of the absorbance respect to time or potential, has been scarcely used to study and understand redox reactions of several systems [35-37] and to characterize thin electrochromic films [38]. Furthermore, the quantification of acetylsalicylic acid in presence of AA and UA has been reported using a modified electrode and the derivative of the absorbance respect to potential at a characteristic wavelength as quantitative signal [39].

In this work, we propose the use of the derivative of the absorbance respect to the wavelength as analytical signal in time-resolved experiments where multiple spectra were acquired during a cyclic voltammograms (CV). The whole set of derivative spectroelectrochemical data has been statistically analyzed using multivariate chemometric tools that help to unravel this complex set of data. In particular, in this work, parallel factor analysis (PARAFAC) [40-42] has been chosen because this set of data is intrinsically trilinear; for each sample studied with the three analytes at different concentrations, absorbance depends both on the potential applied and on the wavelength.

To sum up, the main objective of our work is the development of a new methodology that combines the evolution of the derivative of the absorbance with respect to the wavelength during an electrochemical experiment with PARAFAC as statistical tool to 
solve complex problems. This methodology is demonstrated, as a proof of concept, with the simultaneous determination of DA, AA and UA using commercial SPEs without any modification.

\section{MATERIALS AND METHODS}

\section{Reagents and Materials}

Dopamine (DA, 3-Hydroxytyramine hydrochloride, 99\%, Acros Organics), ascorbic acid (AA, L(+)-Ascorbic acid, ACS reagent, Acros Organics), uric acid (UA, 99+\%, Acros Organics) and $\mathrm{HCl}(37 \%, \mathrm{VWR})$ were used as received without further purification. All solutions were daily prepared using high-quality water $(18.2 \mathrm{M} \Omega \mathrm{cm}$ resistivity at 25 oC, Milli-Q Direct 8, Millipore). All experiments were performed at room temperature using SPEs (DRP-110, Metrohm-DropSens). Each SPE has a $4 \mathrm{~mm}$ diameter disk screenprinted carbon working electrode (WE), a carbon counter electrode (CE) and a silver pseudoreference electrode (RE).

\section{Instrumentation}

SEC experiments were carried out using a customized SPELEC instrument (MetrohmDropSens) controlled by DropView SPELEC software (Metrohm-DropSens).

\section{Experimental Setup}

SEC experiments in parallel configuration under semi-infinite diffusion regime were performed using previous devices developed by our research group [21, 23]. Two bare optical fibers (100 $\mu \mathrm{m}$ in diameter, Ocean Optics) were perfectly aligned face to face and fixed with nail polish on the working electrode to carry out SEC experiments in parallel configuration. The distance between the ends of the two bare optical fibers 
defined the optical path length. Therefore, it is very easy to change the distance between the fibers, which allows us to study compounds with very different molar absorption coefficients. In order to perform the corresponding experiments, a solution drop $(100 \mu \mathrm{L})$ was placed on the electrode, covering the three-electrode system and the ends of the bare optical fibers. Finally, one of the optical fibers was connected to the light source and the other one to the spectrometer.

On the other hand, SEC experiments in parallel arrangement and under thin-layer diffusion regime were carried out with the same device, but placing a transparent quartz plate on the fibers and the working electrode to ensure the thin layer regime [24]. A transparent plate is used because it allows us to follow the process. Two bare optical fiber pieces were placed at the ends of the electrode support to ensure that the quartz plate is completely parallel to the electrode surface, ensuring that the distance between the electrode and the quart plate is always the same. The volume of the solution drop is $40 \mu \mathrm{L}$ and it has to be added on the electrode before placing the quartz plate. The thickness of the thin-layer can be controlled by the diameter of the fibers used. In this case, it is approximately of $140 \mu \mathrm{m}$ (100 $\mu \mathrm{m}$ corresponds to the diameter of the fiber and $20 \mu \mathrm{m}$ to its cladding/coating materials) [24]. Thin-layer SEC has important advantages over semi-infinite or normal configuration measurements. It is characterized by a high sensitivity due to the long optical pathway, it is possible to completely electrolyze the whole solution, and the use of optical transparent electrodes is not required.

It should be highlighted that in each experiments the spectrum of the initial solution was taken as reference spectrum. The same SPE with the optical fibers fixed on it has 
been used to measure all solutions in each calibration model. Optical signals have been filtered using a Savitzky-Golay filter that allows obtaining the derivative signals.

\section{PARAFAC analysis}

Matlab R2018a was used to carry out the PARAFAC analysis, using the N-way toolbox developed by Rasmus Bro [43].

\section{RESULTS AND DISCUSSION}

Comparison between UV/Vis absorption SEC under semi-infinite diffusion and thinlayer regime.

In order to demonstrate the better performance and higher sensitivity of parallel configuration under thin-layer diffusion regime with respect to semi-infinite diffusion regime some characteristic experiments are described below. UV/Vis absorption SEC experiments in parallel configuration under both regimes and choosing UA as model analyte were performed. Cyclic voltammograms of UA $1 \cdot 10^{-4} \mathrm{M}$ in acidic medium $(\mathrm{HCl}$ $0.1 \mathrm{M}$ ) were recorded between $+0.30 \mathrm{~V}$ and $+0.80 \mathrm{~V}$ at $0.005 \mathrm{~V} \mathrm{~s}^{-1}$ and, simultaneously, the evolution and changes of the UV/Vis absorption spectra were registered every 200 ms. The oxidation of UA shows an irreversible anodic peak around $+0.60 \mathrm{~V}$ in the two diffusion regimes (Fig. 1a). Concomitantly, oxidation of UA shows the decrease of a band at $288 \mathrm{~nm}$, assigned to the consumption of UA during the anodic scan.[44] Fig. 1b shows this process for the experiment performed under thin-layer diffusion regime. 

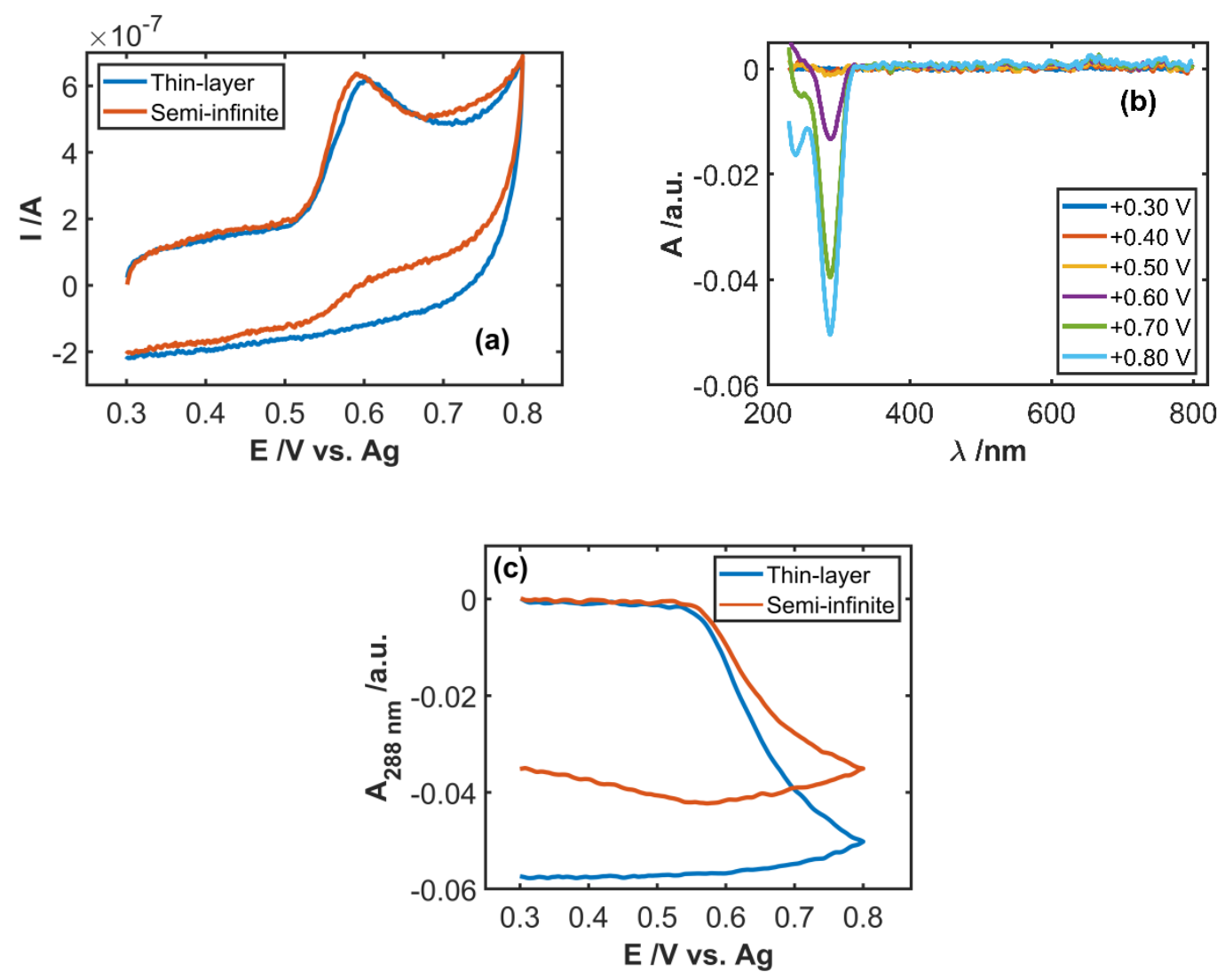

Fig. 1. (a) CVs, (b) spectra evolution during anodic scan in thin-layer diffusion regime, and (c) voltabsorptograms at $288 \mathrm{~nm}$. Solution UA $1 \cdot 10^{-4} \mathrm{M}$ in $\mathrm{HCl} 0.1 \mathrm{M}$. The potential is scanned between $+0.30 \mathrm{~V}$ and $+0.80 \mathrm{~V}$ at $0.005 \mathrm{~V} \mathrm{~s}^{-1}$. Blue lines in (a) and (c) are related to signals in thin-layer diffusion regime. Red lines in (a) and (c) are associated with signals in semi-infinite diffusion regime.

Selecting this UA characteristic wavelength, $288 \mathrm{~nm}$, Fig. 1c depicts the corresponding voltabsorptograms in thin-layer and in semi-infinite diffusion regimes. In both voltabsorptograms it is observed that in the anodic scan the oxidation of UA starts at $+0.55 \mathrm{~V}$, decreasing the absorbance at $288 \mathrm{~nm}$ until the vertex potential, $+0.80 \mathrm{~V}$, due to the consumption of UA. Besides, in the backward scan, absorbance does not recover the starting zero value, due to the irreversible oxidation of UA. However, some differences can be observed in these two voltabsorptograms. On the one hand, in thinlayer diffusion regime, a stationary state is reached during the reduction scan 
indicating that practically all the UA present in the solution has been consumed. Consequently, in the backward scan the absorbance reaches a constant value related to the amount of UA consumed during the oxidation. Meanwhile, in semi-infinite diffusion regime, a small increase of absorbance from $+0.55 \mathrm{~V}$ onwards is observed during the cathodic scan. It is due to the diffusion of UA from the bulk solution to the area sampled by the optical fibers at potentials higher than the oxidation potential. Furthermore, another significant difference is observed in Fig. 1c. The absorbance value achieved in thin-layer is ca. $36 \%$ higher than the one obtained in semi-infinite configuration because the diffusion is limited in the thin-layer arrangement and the electrolysis of all UA occurs in the solution sampled by the optical fibers. This aspect confers a higher sensitivity to the thin-layer diffusion regime configuration.

Furthermore, to check the improvement in sensitivity when performing the experiments in thin-layer regime rather than in semi-infinite diffusion, two calibration models were constructed using both configurations. A set of calibration samples with concentrations of UA between $1 \cdot 10^{-5}$ and $1 \cdot 10^{-4} \mathrm{M}$ in $0.1 \mathrm{M} \mathrm{HCl}$ were measured by UV/Vis absorption SEC performing voltammetric and voltabsorptometric measurements between +0.30 and $+0.80 \mathrm{~V}$ at $0.005 \mathrm{~V} \mathrm{~s}^{-1}$, in both regimes. This range of concentrations was chosen because it is the concentration interval in which a linear correlation is achieved for both configurations. For each diffusion regime, two linear calibration models were constructed with the spectroelectrochemical data, one correlating the anodic peak current of the CV with UA concentration, while the other one correlates the absorbance values at $288 \mathrm{~nm}$ and at the vertex potential $(+0.80 \mathrm{~V})$ with the UA concentration. The main statistical indicators used to assess the quality of each calibration model, such as the coefficient of determination $\left(R^{2}\right)$, the sensitivity 
and the standard deviation of residuals of the linear regression $\left(S_{y x}\right)$ are summarized in Table 1.

Table 1. Regression parameters obtained for the determination of UA by UV/Vis-SEC in thin-layer and semi-infinite diffusion regimes.

\begin{tabular}{|c|c|c|c|c|}
\hline \multicolumn{2}{|c|}{ Analysis method ${ }^{(1)}$} & $R^{2,(2)}$ & Sensitivity ${ }^{(3)}$ & $S_{y x}{ }^{(4)}$ \\
\hline \multirow{2}{*}{ Thin-layer } & $I_{p}$ vs. [UA] & 0.9900 & $3.63 \cdot 10^{-3} \mathrm{~A} / \mathrm{M}$ & $1.33 \cdot 10^{-8}$ \\
& $\mathrm{~A}_{288 \mathrm{~nm},+0.80 \text { v vs. [UA] }} 0.9870$ & -441.83 a.u./M & $1.87 \cdot 10^{-3}$ \\
\hline \multirow{2}{*}{ Semi-infinite } & $\mathrm{I}_{\mathrm{p}}$ vs. [UA] & 0.9903 & $3.70 \cdot 10^{-3} \mathrm{~A} / \mathrm{M}$ & $1.29 \cdot 10^{-8}$ \\
& $\mathrm{~A}_{288 \mathrm{~nm},+0.80 \text { v vs. [UA] }} 0.9869$ & -341.02 a.u./M & $1.50 \cdot 10^{-3}$ \\
\hline
\end{tabular}

(1) Concentration range: UA $10^{-5}-10^{-4} \mathrm{M}$ in $\mathrm{HCl} 0.1 \mathrm{M}$; electrochemical method correlates anodic peak current with UA concentration; spectroscopic method correlates absorbance at $288 \mathrm{~nm}$ and $+0.80 \mathrm{~V}$ with UA concentration. All data are obtained during the same experiment and at the same time/potential.

(2) Coefficient of determination.

(3) Slope of the linear regression model.

(4) Standard deviation of residuals.

From the electrochemical data, it can be concluded that the diffusion regime selected does not imply any difference; all statistical parameters are similar, even the sensitivity. However, from the spectroscopic data obtained simultaneously to the electrochemical ones, one remarkable difference is found. The sensitivity in thin-layer experiments is $30 \%$ higher than in semi-infinite diffusion regime. The facts that in thinlayer regime the total electrolysis of the analyte can be achieved and that the diffusion from the bulk solution is limited determine this increase in the sensitivity, making UV/Vis absorption SEC in thin-layer configuration more suitable for quantitative analysis.

Simultaneous determination of DA, AA and UA with derivative UV/Vis absorption thin-layer SEC. 
Once demonstrated the highest sensitivity of the thin-layer configuration from a quantitative point of view, the main objective of this work is to prove the great possibilities that UV/Vis absorption thin-layer SEC offers in the determination of very complex mixtures, from both the electrochemical and spectroscopic point of view, as is the case of DA, AA and UA in acid medium. As was explained above, voltammetric and voltabsorptometric experiments have been performed simultaneously to study and quantify the electrochemical oxidation of these analytes. In this case, the potential was swept between 0.00 and $+0.90 \mathrm{~V}$ at $0.005 \mathrm{~V} \mathrm{~s}^{-1}$, and the integration time in the acquisition of spectra was 400 ms. Therefore, with this purpose, different calibration samples were prepared to construct a calibration model able to predict the concentration of test samples (Table S1, in Electronic Supplementary Material, ESM). All solutions were prepared in $\mathrm{HCl} 0.1 \mathrm{M}$ changing the concentration of the three analytes between $1 \cdot 10^{-5}$ and $1 \cdot 10^{-4} \mathrm{M}$. The concentration of each compound in each sample was randomly chosen.

Each experiment provides an electrochemical signal, a CV, and the simultaneous evolution of the spectroscopic signal along the CV. Fig. 2a shows the CVs of aqueous solutions in $\mathrm{HCl} 0.1 \mathrm{M}$ of DA $\left(1 \cdot 10^{-4} \mathrm{M}\right), \mathrm{AA}\left(1 \cdot 10^{-4} \mathrm{M}\right), \mathrm{UA}\left(1 \cdot 10^{-4} \mathrm{M}\right)$ and of a solution mixture of the three molecules, which corresponds with the experiment E05 of Table S1 (in ESM). 

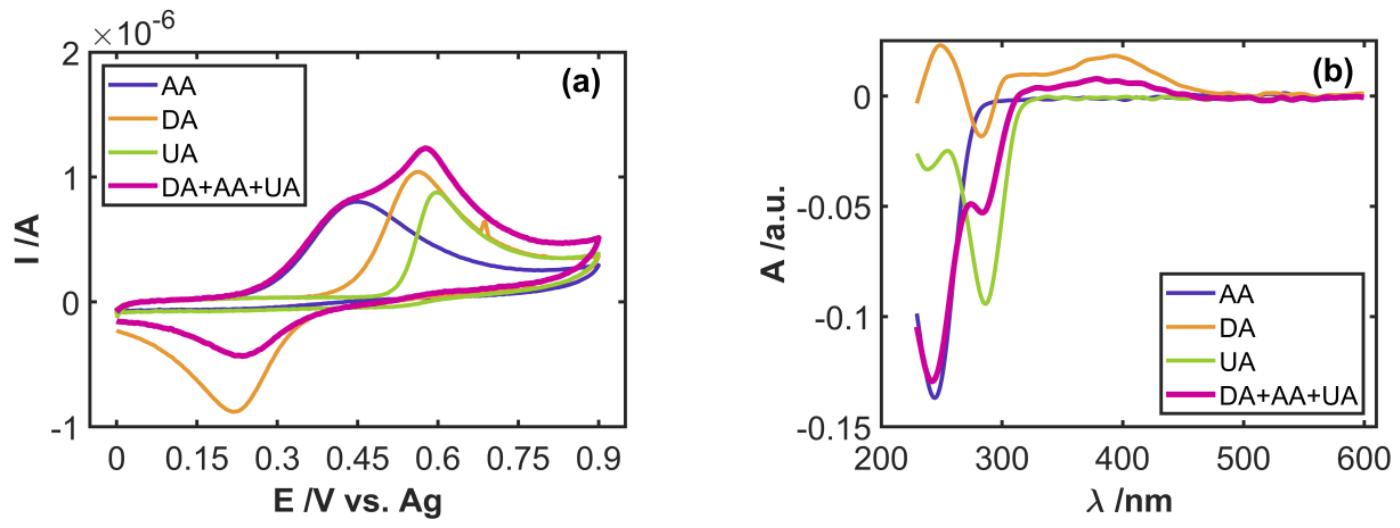

Fig. 2. (a) CVs and (b) absorption spectra registered at the vertex potential (+0.90 V). Spectroelectrochemical responses recorded between $0.00 \mathrm{~V}$ and $+0.90 \mathrm{~V}$ at $0.005 \mathrm{~V} \mathrm{~s}^{-1}$ of $A A 1 \cdot 10^{-4} \mathrm{M}$ (blue line), DA $1 \cdot 10^{-4} \mathrm{M}$ (orange line), UA $1 \cdot 10^{-4} \mathrm{M}$ (green line) and of a complex mixture of $D A 5 \cdot 10^{-5} \mathrm{M}+A A 9 \cdot 10^{-5} \mathrm{M}+U A 4 \cdot 10^{-5} \mathrm{M}$ (magenta line) in $\mathrm{HCl} 0.1$ M.

These CVs show the redox behavior of these species alone and in a mixture of them. In acid media, AA is electrochemically oxidized to dehydroascorbic acid (DHA) by transfer of two protons and two electrons in a reversible electron transfer process coupled to an irreversible chemical reaction where DHA yields to an electroinactive product after solvation $[45,46]$. Consequently, in the CV of AA (Fig. 2a, blue line) it is observed only one anodic peak at $+0.45 \mathrm{~V}$ related to the electrogeneration of $\mathrm{DHA}$. At $\mathrm{pH}=1(\mathrm{HCl} 0.1$ $M), D A$ is oxidized to dopaminequinone (DAQ) in a two protons and two electrons quasi-reversible process [15], so that its CV shows an anodic and a cathodic peak at $+0.56 \mathrm{~V}$ and $+0.22 \mathrm{~V}$, respectively (Fig. 2a, orange line). Lastly, UA shows an irreversible behavior as was stated in Fig. 1, with one anodic peak at $+0.60 \mathrm{~V}$ (Fig. 2a, green line). The electrochemical oxidation of UA yields di-imine species by a two protons and two electrons reversible process. This di-imine species in aqueous solution is unstable and after solvation it is transformed into an imine-alcohol, also unstable that is hydrated to 
give uric acid-4,5-diol $[44,47]$. The anodic peaks related to the oxidation of these three molecules emerge at very similar potentials, which involves that in mixture solutions of the three molecules the overlapping is really high. As can be observed in Fig. $2 a$ (magenta line), only one anodic peak at $+0.58 \mathrm{~V}$ with a shoulder at $+0.45 \mathrm{~V}$ is observed, being very difficult to determine any of these molecules in presence of the others using only the electrochemical signal.

However, it is more difficult to find two compounds with the same electrochemical and spectroscopic behavior. Fig. $2 b$ shows the absorption spectra of each solution at $a$ potential of $+0.90 \mathrm{~V}$ registered during the cyclic voltammetric experiment. In all cases the spectrum of the initial solution with the corresponding analyte (DA, UA, AA or the mixture of them) was taken as reference in the absorptometric measurements. The spectrum of the AA solution (Fig. $2 \mathrm{~b}$, blue line) shows a single negative absorption band at $245 \mathrm{~nm}$, related to the consumption of AA during the anodic scan [21]. In the same way, the electrooxidation of UA yields an absorption spectrum with a negative band at $288 \mathrm{~nm}$ (Fig. 2b, green line), related to the consumption of UA [44]. Finally, the spectrum related to the oxidation of $\mathrm{DA}$ in $\mathrm{HCl} 0.1 \mathrm{M}$ (Fig. 2b, orange line) shows two positive absorption bands peaking at 395 and $250 \mathrm{~nm}$, characteristics of the o-quinone group of the DAQ electrogenerated [48]. Additionally, a negative absorption band at $280 \mathrm{~nm}$ is observed related to the $L_{a}$ and $L_{b}$ transitions of the DA consumed during this oxidation process [49]. As can be appreciated in Fig. $2 b$ (magenta line), the absorption spectrum related to the oxidation of a mixture solution of the three analytes shows a number of overlapping bands related to all the molecules consumed and the DAQ, the only oxidation product observed in these experiments. From these signals it can be concluded that the spectroscopic quantification of these compounds is also very 
complicated due to the great overlapping of the absorption bands between 200 and $310 \mathrm{~nm}$. Only DA could be detected in the presence of AA and UA using the spectroscopic signals from the UV/Vis absorption spectroelectrochemical data, using the absorption band peaking at $390 \mathrm{~nm}$.

Nevertheless, the determination of all these molecules is essential, due to the fundamental role that they play in the human metabolism. For this purpose, a multivariate technique using PARAFAC and based on the derivative of the spectroscopic response is proposed, due to its capability to solve complex matrixes without the need to carry out any pre-treatment or modification of the electrode surface. The reason of choosing the derivative of absorbance with respect to the wavelength as analytical signal in the quantification of mixtures of DA, AU and AA in $\mathrm{HCl} 0.1 \mathrm{M}$ medium is because this transformation of the optical signals allows a better discrimination of all absorptometric changes occurring during the voltammetric experiment. Fig. 3a shows the non-derivative spectral changes registered during the oxidation of a mixture of these three molecules. As can be observed, a main absorption band decreases at $243 \mathrm{~nm}$ overlapped with a second absorption band peaking at $285 \mathrm{~nm}$, while a small band increases around $390 \mathrm{~nm}$. From these spectra is extremely difficult to distinguish the evolution of the different molecules during this potential cycle. However, with the derivative of each spectrum with respect to the wavelength ( $d A / d \lambda$, Fig. $3 b)$, some differences can be appreciated that will help to discriminate the evolution of each molecule. The comparison of the derivative of the spectrum at the vertex potential of pure samples with only one molecule and the derivative of the spectrum at the same potential of the solution mixture of the three 
compounds displays the possibilities that this transformation of the absorptometric signals offers (Fig. 3c).
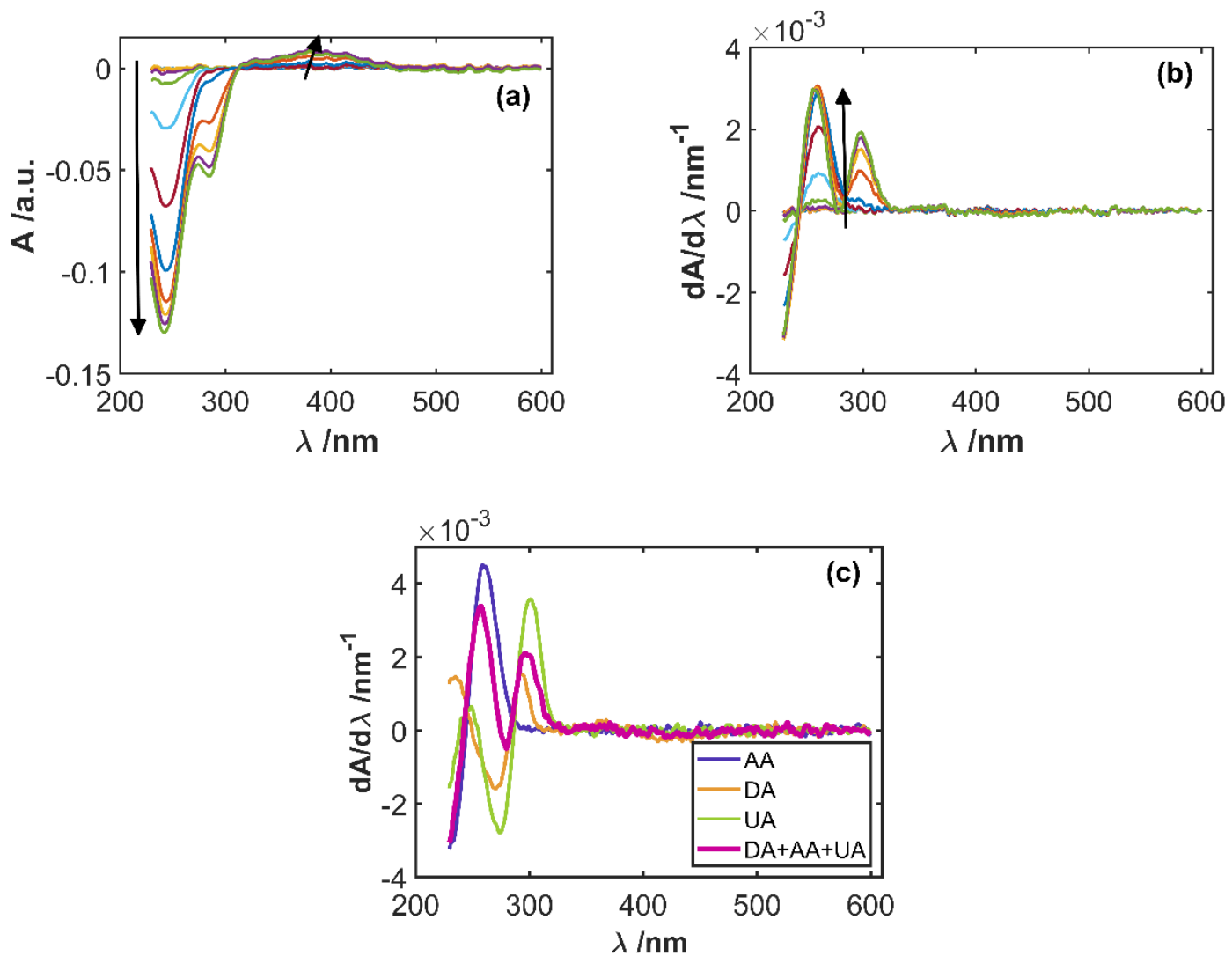

Fig. 3. Evolution of (a) absorption spectra and (b) derivative spectra with respect to the wavelength between $0.00 \mathrm{~V}$ and $+0.90 \mathrm{~V}$ at $0.005 \mathrm{~V} \mathrm{~s}^{-1}$ of a complex mixture of $D A$ $5 \cdot 10^{-5} \mathrm{M}+\mathrm{AA} 9 \cdot 10^{-5} \mathrm{M}+\mathrm{UA} 4 \cdot 10^{-5} \mathrm{M}$ in $\mathrm{HCl} 0.1 \mathrm{M}$. (c) Derivative spectra with respect to the wavelength at the vertex potential (+0.90 V) of $A A 1 \cdot 10^{-4} \mathrm{M}$ (blue line), DA $1 \cdot 10^{-4}$ $M$ (orange line), UA $1 \cdot 10^{-4} \mathrm{M}$ (green line) and of a solution mixture of $D A 5 \cdot 10^{-5} \mathrm{M}+A A$ $9 \cdot 10^{-5} \mathrm{M}+U A 4 \cdot 10^{-5} \mathrm{M}$ (magenta line) in $\mathrm{HCl} 0.1 \mathrm{M}$.

After the interest and advantages that the derivative of the absorbance with respect to the wavelength $(d A / d \lambda)$ has in the analysis of mixtures of molecules in a solution, we propose the use of these signals to resolve and quantify mixtures of $A A, D A$ and UA with PARAFAC. The matrix used to construct the calibration model with PARAFAC 
consisted of a three-dimensional data matrix with the values of $d A / d \lambda$ from $+0.28 \mathrm{~V}$ in the forward scan to the end of the backward scan $(0.00 \mathrm{~V})$ and between 235 and 550 $\mathrm{nm}$ for each sample prepared and measured (Table S1, in ESM). The selected potential window corresponds to the potentials at which some oxidation or reduction were observed both in the CV and in the spectra. Furthermore, the chosen wavelength window matched with the UV/Vis spectral region where significant changes of $d A / d \lambda$ took place. Hence, regions without analytical information about the mixture of $A A, D A$ and UA were excluded. The matrix dimension is defined by the values of $d A / d \lambda$ at 761 potentials $\times 397$ wavelengths $\times 14$ concentrations, and corresponds with up to 4229638 values of $d A / d \lambda$ to construct the PARAFAC model. According to the three compounds present in the mixture (DA, AA and UA), three components were selected, one for each molecule. The outputs of the model were obtained after 42 iterations with a corcondia value of $89.4 \%$. This value is the core consistency diagnostic that gives a clear-cut answer to whether a model is appropriate or not. When the PARAFAC model is valid, the corcondia value is close to $100 \%$ [50].

Fig. $4 \mathrm{a}$ and Fig. $4 \mathrm{~b}$ represent the loadings of the three components, which can be easily ascribed to one of the three molecules (DA, AA and UA), with respect to the range of potentials selected and to the range of wavelengths chosen to obtain the PARAFAC model, respectively. As can be seen in Fig. 4a, the plot of the loadings vs. the potential applied shows that the component ascribed to AA shows its irreversible oxidation at lower potentials, from $+0.30 \mathrm{~V}$ onwards. Next, the component assigned to DA, indicates the reversible oxidation of $\mathrm{DA}$, from $+0.45 \mathrm{~V}$ onwards. Finally, the last component is related to the irreversible oxidation of UA that occurs from $+0.55 \mathrm{~V}$ onwards. 

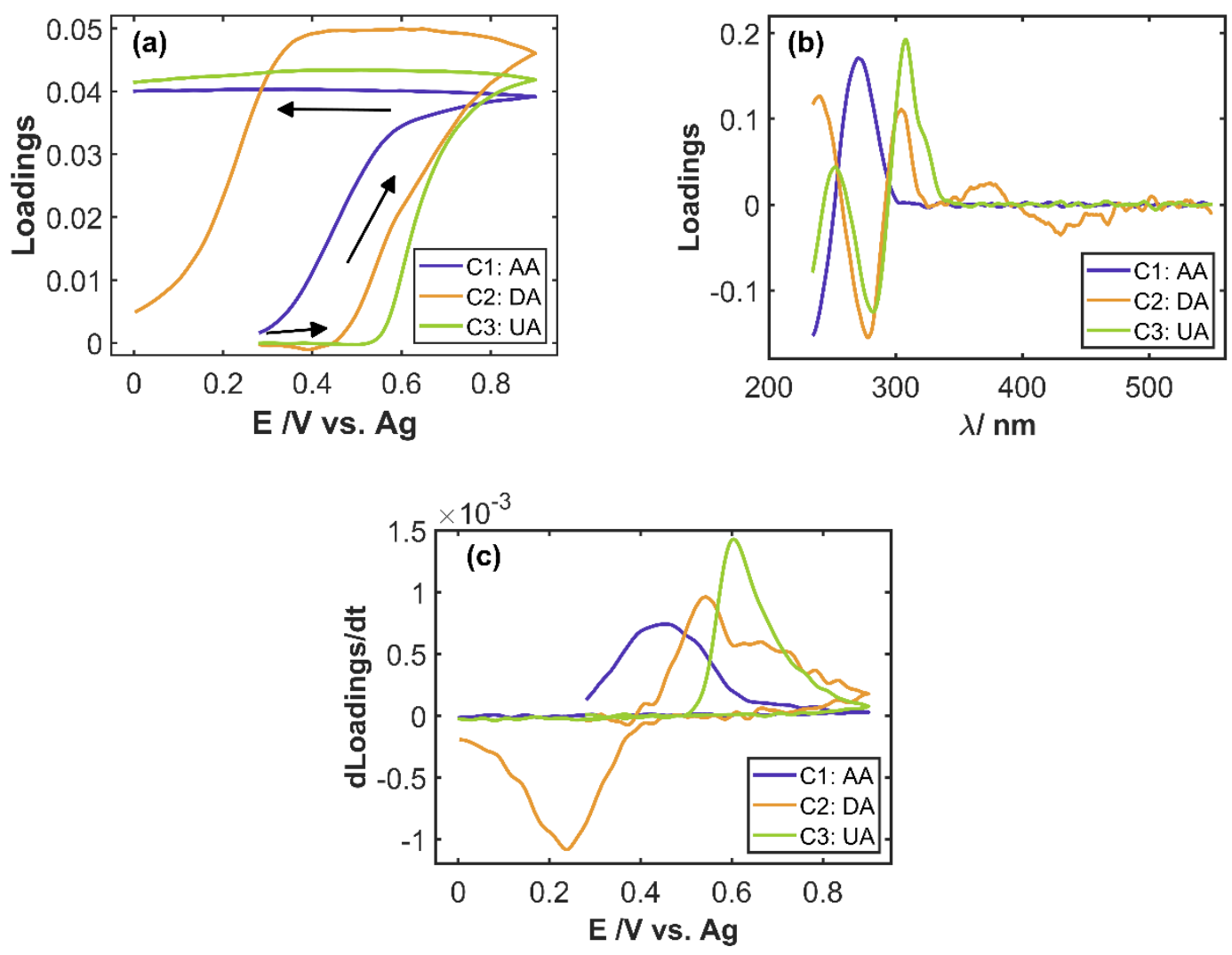

Fig. 4. Loadings of each component versus (a) the potential and (b) the wavelength, after PARAFAC analysis. (c) Derivative of loadings with respect to time for each component. Blue line is the signal of the first component related to $A A$; orange line is the signal of the second component related to DA; and green line is the signal of the third component related to $\cup A$.

These processes of sequential oxidation of the three molecules are better observed in the plot of the derivative of the loadings with respect to time (Fig. 4c), with anodic peaks at $+0.45 \mathrm{~V},+0.54 \mathrm{~V}$ and $+0.60 \mathrm{~V}$ related to $\mathrm{AA}, \mathrm{DA}$ and $\mathrm{UA}$, respectively. The comparison of the three signals in Fig. $4 \mathrm{c}$ with the corresponding CVs registered with solutions prepared with only one of the molecules shows a really high similarity (Fig. S1, in ESM), taking into account that the loadings have been exclusively obtained from absorptometric values. These similarities can also be appreciated between the signals in Fig. 4b obtained from PARAFAC model and the derivative of the absorbance with 
respect to the wavelength of solutions with only one of the molecules (Fig. S2, in ESM).

Therefore, we can conclude that the PARAFAC model constructed with the values of the derivative of absorbance with respect to the wavelength allows the deconvolution of the optical signals related to each of the three molecules tested, i.e., AA, DA and UA.

Once the optical signals are deconvolved, the determination of each molecule can be carried out. With this objective, three calibration curves were constructed using a linear least squares method, one for each molecule, correlating the values of the loadings for each component with the concentration of each specific molecule in the different mixtures tabulated in Table S1 (in ESM). Fig. S3 (in ESM) and Table 2 show the figures of merit of these calibration models. In these calibration models, the sample E05, used as test sample, was excluded.

Table 2. Regression parameters obtained for the determination of $A A, D A$ and $U A$ in mixtures by UV/Vis-SEC in thin-layer regime with the loadings of the PARAFAC model.

\begin{tabular}{|c|c|c|c|c|c|c|}
\hline Molecule & Calibration model & $\mathbf{R}^{\mathbf{2},(2)}$ & $\begin{array}{c}\text { Sensitivity } \\
\mathbf{( M}^{-1} \mathbf{)}\end{array}$ & $\mathbf{S}_{\mathbf{y x}} \mathbf{( 4 )}^{(\mathbf{3})}$ & $\begin{array}{c}\mathbf{R S D}_{\text {slope }^{(5)}} \\
\mathbf{( \% )}\end{array}$ & $\begin{array}{c}\text { LOD }^{(\mathbf{6})} \\
\text { (M) }\end{array}$ \\
\hline AA & Loadings C1 vs. [AA] & 0.9976 & 5582.7 & $1.09 \cdot 10^{-2}$ & 0.3 & $0.6 \cdot 10^{-5}$ \\
\hline DA & Loadings C2 vs. [DA] & 0.9941 & 1920.7 & $0.66 \cdot 10^{-2}$ & 8.7 & $1.6 \cdot 10^{-5}$ \\
\hline UA & Loadings C3 vs. [UA] & 0.9970 & 5301.6 & $1.31 \cdot 10^{-2}$ & 11.8 & $1.2 \cdot 10^{-5}$ \\
\hline
\end{tabular}

(1) Correlation between the loadings obtained from the PARAFAC model for the three components and the concentrations of AA, DA or UA in samples tabulated in Table S1 (in ESM), except E05.

(2) Coefficient of determination.

(3) Slope of the linear regression model.

(4) Standard deviation of residuals.

(5) Relative standard deviation of the slope ( $n=3)$.

(6) Limit of detection.

High coefficients of determination $\left(R^{2}\right)$ and low values of the standard deviation of residuals of the regressions $\left(S_{y x}\right)$ were obtained for the three linear calibration models 
constructed for the molecules, indicating the suitable deconvolution of the raw signals with the PARAFAC model obtained.

The repeatability of the procedure was evaluated replicating some samples three times. These replicated samples (E06, E09 and E10) can be found in Table S1 (in ESM). The repeatability was evaluated for each calibration model constructed for each molecule, obtaining \%RSD values lower than $5.5 \%$ in all cases, indicating that the procedure provides good repeatability.

The reproducibility of the procedure was demonstrated performing three PARAFAC models with data collected in three different days using each day a different SPE with the optical fibers fixed on it. The set of experiments was always the same as shown in Table S1 (in ESM). The values of the relative standard deviation of the slope indicate a high reproducibility (Table $2, \mathrm{RSD}_{\text {slope }}$ values). Lastly, the limits of detection were calculated, with values for the three molecules lower than $16 \mu \mathrm{M}$.

Finally, the concentration of each molecule in a test sample (sample E05 in Table S1, in ESM) was estimated with each one of the linear calibration models constructed in three different days (Table 3). The estimated concentration of each molecule shows values with a relative error lower than 3\% (AA and UA) and 9\% (DA). These values indicate that the procedure followed and the linear models developed allow a suitable assessment with a high precision of the unknown concentrations of $A A, D A$ and UA in a test mixture. Furthermore, the values of the relative standard deviation of the estimated concentrations ( $R \mathrm{RD}_{\text {conc }}$ ) for each molecule with each one of the three linear calibration models are lower than $5 \%$, denoting a high reproducibility. 
Table 3. Estimation of the concentration of $A A, D A$ and UA in a test sample.

\begin{tabular}{|c|c|c|c|c|c|}
\hline Molecule & [Real] $^{(1)}(\mathrm{M})$ & Cestimated $^{(2)}(\mathrm{M})$ & $\mathrm{Cl}^{(3)}(\mathrm{M})$ & R.E. ${ }^{(4)}(\%)$ & $\mathrm{RSD}_{\text {conc }}{ }^{(5)}(\%)$ \\
\hline AA & $9 \cdot 10^{-5}$ & $9.1 \cdot 10^{-5}$ & {$[8.3-9.9] \cdot 10^{-5}$} & 0.8 & 3.6 \\
\hline DA & $5 \cdot 10^{-5}$ & $4.6 \cdot 10^{-5}$ & {$[4.1-5.1] \cdot 10^{-5}$} & 8.1 & 4.1 \\
\hline UA & $4 \cdot 10^{-5}$ & $3.9 \cdot 10^{-5}$ & {$[3.5-4.3] \cdot 10^{-5}$} & 2.0 & 4.3 \\
\hline
\end{tabular}

(1) Real concentration of each molecule in the test sample (E05, Table S1, in ESM).

(2) Media of the estimated concentration of each molecule of the test mixture sample with the constructed linear calibration models (Table 2).

(3) Confidence interval, $\alpha=0.05$.

(4) Relative error calculated as $/ C_{\text {estimated }}-C_{\text {real }} / \cdot 100 / C_{\text {real }}$.

(5) Relative standard deviation of the concentration $(n=3)$.

The results presented, both in the deconvolution of the raw spectroelectrochemical signals and in the simultaneous quantification of DA, AA and UA in a mixture, demonstrate that the strategy proposed has been successfully implemented. The combination of derivative UV/Vis absorption SEC with thin-layer regime measurements and multivariate chemometric tools such as PARAFAC has allowed us to determine at the same time these three molecules, which show high overlapping both in the electrochemical and in the absorptometric signals.

\section{CONCLUSIONS}

The analysis of high overlapping signals where three different molecules are responsible of the changes of current or absorbance in spectroelectrochemical experiments is highly complicated. However, the development of strategies that allow the researchers to know the exact contribution of each molecule in a sample to the signals studied is highly interesting. In this work, the combination of derivative UV/Vis 
absorption spectroelectrochemistry data and suitable multivariate chemometric tools has allow us to successfully achieve this objective.

Thin-layer experiments, where the diffusion of reactants and products is limited, have been very helpful to improve the sensitivity of the methodology proposed. Longoptical path-way experiments, where the optical path way is delimited by the distance between the two optical fibers, have also contributed to increase this sensitivity.

The oxidation of AA, DA, and UA in mixture samples supplies high overlapping signals both in the CVs and in the absorption spectra. The best strategy to study this kind of samples has demonstrated to be the use of the derivative of the absorbance with respect to the wavelength because it allows a much better differentiation of the range of potentials at which each molecule is oxidized. Additionally, the use of PARAFAC, a multivariate chemometric tool useful in these kinds of problems due to the trilinear character of spectroelectrochemical data, has allowed to successfully deconvolve the pristine spectroelectrochemical signals and to quantify DA, AA and UA simultaneously, yielding to micromolar limits of detection. The sensitivity of the method is clearly dependent on the molecule studied, being in this case much more sensitive to AA and UA than to DA.

Nevertheless, instrumental improvements should help to obtain better limits of detection in the near future, making SEC a very interesting technique for quantitative analysis because of its intrinsic trilinear character. Developments in electrochemistry instrumentation helped in the past to improve limits of detection and, in the same way, developments in optical equipment should help SEC. Detectors with better signalto-noise ratio, more stable light sources and better optical fibers could help to highly 
improve limits of detection, allowing applying the methodology presented in this work to more complex problems.

The results presented here show only one of the multiple possibilities that the synergy between spectroelectrochemical data and chemometric tools has in solving complex chemical problems, avoiding tough and time-consuming strategies of optimization and fabrication of chemically modified electrodes.

\section{ACKNOWLEDGEMENTS}

Authors acknowledge the financial support from Ministerio de Economía y Competitividad (Grants CTQ2017-83935-R-AEI/FEDER, UE), Junta de Castilla y León (Grant BU297P18) and Ministerio de Ciencia, Innovación y Universidades (RED2018102412-T). F.O. thanks its contract funded by Junta de Castilla y León, the European Social Fund and the Youth Employment Initiative. J.G.R. thanks Ministerio de Economía y Competitividad for his postdoctoral contract (CTQ2017-83935-R AEI/FEDER, UE).

\section{CONFLICT OF INTEREST}

Fabiola Olmo has a contract funded by Junta de Castilla y León, the European Social Fund and the Youth Employment Initiative. Jesús Garoz-Ruiz acknowledges his postdoctoral research grant from Ministerio de Economía y Competitividad (CTQ201783935-R AEI/FEDER, UE). Alvaro Colina is the main researcher of the project CTQ201783935-R-AEI/FEDER from the Spanish Ministerio de Economía y Competitividad. Aránzazu Heras Vidaurre is the main researcher of the project BU297P18 from the Spanish Junta de Castilla y León. Alvaro Colina and Aránzazu Heras participate in the research network RED2018-102412-T from the Spanish Ministerio de Ciencia, Innovación y Universidades. 


\section{REFERENCES}

1. González-Diéguez N, Colina A, López-Palacios J, Heras A (2012) Spectroelectrochemistry at screen-printed electrodes: Determination of dopamine. Anal Chem 84:9146-9153. https://doi.org/10.1021/ac3018444

2. Kim Y-R, Bong S, Kang Y-J, Yang Y, Mahajan RK, Kim JS, Kim H (2010) Electrochemical detection of dopamine in the presence of ascorbic acid using graphene modified electrodes. Biosens Bioelectron 25:2366-2369. https://doi.org/10.1016/j.talanta.2012.05.013

3. Jin GP, Peng X, Ding YF (2008) The electrochemical modification of clenbuterol for biosensors of dopamine, norepinephrine, adrenalin, ascorbic acid and uric acid at paraffin-impregnated graphite electrode. Biosens Bioelectron 24:10311035. https://doi.org/10.1016/j.bios.2008.06.028

4. Zou C, Zhong J, Li S, Wang H, Wang J, Yan B, Du Y (2017) Fabrication of reduced graphene oxide-bimetallic PdAu nanocomposites for the electrochemical determination of ascorbic acid, dopamine, uric acid and rutin. J Electroanal Chem 805:110-119. https://doi.org/10.1016/j.jelechem.2017.10.020

5. Huertas I, Jesús S, Lojo JA, García-Gómez FJ, Cáceres-Redondo MT, Oropesa-Ruiz JM, Carrillo F, Vargas-Gonzalez L, Rodríguez JFM, Gómez-Garre P, García-Solís D, Mir P (2017) Lower levels of uric acid and striatal dopamine in non-tremor dominant Parkinson's disease subtype. PLoS One 12:1-9. https://doi.org/10.1371/journal.pone.0174644

6. Baig N, Rana A, Kawde AN (2018) Modified electrodes for selective voltammetric 
detection of biomolecules. Electroanalysis 30:2551-2574. https://doi.org/10.1002/elan.201800468

7. Yang L, Liu D, Huang J, You T (2014) Simultaneous determination of dopamine, ascorbic acid and uric acid at electrochemically reduced graphene oxide modified electrode. Sensors Actuators, B Chem 193:166-172. https://doi.org/10.1016/j.snb.2013.11.104

8. Ping J, Wu J, Wang Y, Ying Y (2012) Simultaneous determination of ascorbic acid, dopamine and uric acid using high-performance screen-printed graphene $\begin{array}{llll}\text { electrode. } & \text { Biosens } & \text { 3ioelectron }\end{array}$ https://doi.org/10.1016/j.bios.2012.01.016

9. Tukimin N, Abdullah J, Sulaiman Y (2018) Electrodeposition of poly(3,4ethylenedioxythiophene)/reduced graphene oxide/manganese dioxide for simultaneous detection of uric acid, dopamine and ascorbic acid. J Electroanal Chem 820:74-81. https://doi.org/10.1016/j.jelechem.2018.04.065

10. Dinesh B, Vilian ATE, Kwak CH, Huh YS, Saraswathi R, Han YK (2019) The facile and simple synthesis of poly(3,4ethylenedioxythiophene) anchored reduced graphene oxide nanocomposite for biochemical analysis. Anal Chim Acta 1077:150-159. https://doi.org/10.1016/j.aca.2019.05.053

11. Sheng ZH, Zheng XQ, Xu JY, Bao WJ, Wang F Bin, Xia XH (2012) Electrochemical sensor based on nitrogen doped graphene: Simultaneous determination of ascorbic acid, dopamine and uric acid. Biosens Bioelectron 34:125-131. https://doi.org/10.1016/j.bios.2012.01.030

12. Iranmanesh T, Foroughi MM, Jahani S, Shahidi Zandi M, Hassani Nadiki H (2020) 
Green and facile microwave solvent-free synthesis of $\mathrm{CeO}_{2}$ nanoparticledecorated CNTs as a quadruplet electrochemical platform for ultrasensitive and simultaneous detection of ascorbic acid, dopamine, uric acid and acetaminophen. Talanta 207:120318. https://doi.org/10.1016/j.talanta.2019.120318

13. Bagheri H, Pajooheshpour N, Jamali B, Amidi S, Hajian A, Khoshsafar H (2017) A novel electrochemical platform for sensitive and simultaneous determination of dopamine, uric acid and ascorbic acid based on $\mathrm{Fe}_{3} \mathrm{O}_{4}-\mathrm{SnO}_{2}-\mathrm{Gr}$ ternary nanocomposite. Microchem J 131:120-129. https://doi.org/10.1016/j.microc.2016.12.006

14. Huo C, Zhou L, Li Q, Su L, Hou X, Yi K (2018) Preparation of flake hexagonal BN and its application in electrochemical detection of ascorbic acid, dopamine and uric acid. Sensors Actuators B Chem 260:346-356. https://doi.org/10.1016/j.snb.2017.12.208

15. Ibañez D, Garoz-Ruiz J, Heras A, Colina A (2016) Simultaneous UV-visible absorption and Raman spectroelectrochemistry. Anal Chem 88:8210-8217. https://doi.org/10.1021/acs.analchem.6b02008

16. Garoz-Ruiz J, Perales-Rondon J V., Heras A, Colina A (2019) Spectroelectrochemistry of quantum dots. Isr J Chem 59:679-694. https://doi.org/10.1002/ijch.201900028

17. Garoz-Ruiz J, Perales-Rondon JV, Heras A, Colina A (2019) Spectroelectrochemical sensing: current trends and challenges. Electroanalysis 31:1254-1278. https://doi.org/10.1002/elan.201900075 
18. López-Palacios J, Colina A, Heras A, Ruiz V, Fuente L (2001) Bidimensional spectroelectrochemistry. Anal Chem 73:2883-2889. https://doi.org/10.1021/ac0014459

19. Colina A, López-Palacios J, Heras A, Ruiz V, Fuente L (2003) Digital simulation model for bidimensional spectroelectrochemistry. J Electroanal Chem 553:8795. https://doi.org/10.1016/S0022-0728(03)00288-2

20. Garoz-Ruiz J, Heras A, Palmero S, Colina A (2015) Development of a novel bidimensional spectroelectrochemistry cell using transfer single-walled carbon nanotubes films as optically transparent electrodes. Anal Chem 87:6233-6239. https://doi.org/10.1021/acs.analchem.5b00923

21. Garoz-Ruiz J, Heras A, Colina A (2017) Direct Determination of Ascorbic acid in a grapefruit: Paving the way for in vivo spectroelectrochemistry. Anal Chem 89:1815-1822. https://doi.org/10.1021/acs.analchem.6b04155

22. Garoz-Ruiz J, Izquierdo D, Colina A, Palmero S, Heras A (2013) Optical fiber spectroelectrochemical device for detection of catechol at press-transferred single-walled carbon nanotubes electrodes. Anal Bioanal Chem 405:3593-3602. https://doi.org/10.1007/s00216-013-6762-z

23. Garoz-Ruiz J, Guillen-Posteguillo C, Colina A, Heras A (2019) Application of spectroelectroanalysis for the quantitative determination of mixtures of compounds with highly overlapping signals. Talanta 195:815-821. https://doi.org/10.1016/j.talanta.2018.12.002

24. Garoz-Ruiz J, Guillen-Posteguillo C, Heras A, Colina A (2018) Simplifying the assessment of parameters of electron-transfer reactions by using easy-to-use 
thin-layer spectroelectrochemistry devices. Electrochem Commun 86:12-16 . https://doi.org/10.1016/j.elecom.2017.11.001

25. Wang T, Zhao D, Alvarez N, Shanov VN, Heineman WR (2015) Optically transparent carbon nanotube film electrode for thin layer spectroelectrochemistry. Anal Chem 87:9687-9695. https://doi.org/10.1021/acs.analchem.5b01784

26. Wang Y, Jiang H, Tian JJ, He JB (2014) Spectroelectrochemistry of salicylaldehyde oxidation. Electrochim Acta 125:133-140. https://doi.org/10.1016/j.electacta.2014.01.087

27. Murray RW, Heineman WR, O'Dom GW (1967) An optically transparent thin layer cell. Anal Chem 39:1966-1968. https://doi.org/10.1021/ac50156a052

28. Imai K, Okazaki T, Hata N, Taguchi S, Sugawara K, Kuramitz H (2015) Simultaneous multiselective spectroelectrochemical fiber-optic sensor: Demonstration of the concept using methylene blue and ferrocyanide. Anal Chem 87:2375-2382. https://doi.org/10.1021/ac504321u

29. Shtokyo T, Conklin S, Maghasi AT, Richardson JN, Piruska A, Seliskar CJ, Heineman WR (2004) Spectroelectrochemical sensing based on attennuated total internal reflectance stripping voltammetry. 3. Determination of cadmium and copper. Anal Chem 76:1466-1473. https://doi.org/10.1021/ac034829i

30. Chen W, Liu XY, Qian C, Song XN, Li WW, Yu HQ (2015) An UV-vis spectroelectrochemical approach for rapid detection of phenazines and exploration of their redox characteristics. Biosens Bioelectron 64:25-29. https://doi.org/10.1016/j.bios.2014.08.032 
31. Asadpour-Zeynali K, Maryam Sajjadi S, Taherzadeh F (2016) Second order advantage obtained by spectroelectrochemistry along with novel carbon nanotube modified mesh electrode: Application for determination of acetaminophen in Novafen samples. Spectrochim Acta - Part A Mol Biomol Spectrosc 153:674-680. https://doi.org/10.1016/j.saa.2015.09.035

32. Al Alamein AMA, Elwy HM, El-Din SHS (2019) Univariate and multivariate spectrophotometric methods for simultaneous determination of avobenzone and octinoxate in pure form and in cosmetic formulations: A comparative study. Spectrochim Acta - Part A Mol Biomol Spectrosc 206:37-47. https://doi.org/10.1016/j.saa.2018.07.073

33. Moussa BA, El-Zaher AA, Mahrouse MA, Ahmed MS (2016) Resolution of overlapped spectra for the determination of ternary mixture using different and modified spectrophotometric methods. Spectrochim Acta - Part A Mol Biomol Spectrosc 165:127-137. https://doi.org/10.1016/j.saa.2016.04.003

34. Gutiérrez MC (1992) Derivative spectroscopy applied to the determination of alpha and beta acids in hops. J Inst Brew 98:277-281. https://doi.org/10.1002/j.2050-0416.1992.tb01108.x

35. Jin BK, Li L, Huang JL, Zhang SY, Tian YP, Yang JX (2009) IR spectroelectrochemical cyclic voltabsorptometry and derivative cyclic voltabsorptometry. $\quad$ Anal $\quad$ Chem 81:4476-4481. https://doi.org/10.1021/ac9003634

36. Xie Q, Wei W, Nie L, Yao S (1993) Derivative linear sweep and derivative cyclic voltabsorptometry of the long-path-length spectroelectrochemical cell: The 
single reversible electrode reaction. J Electroanal Chem 348:29-47. https://doi.org/10.1016/0022-0728(93)80121-W

37. Cheng WX, Jin BK, Huang P, Cheng LJ, Zhang SY, Tian YP (2013) Investigation on

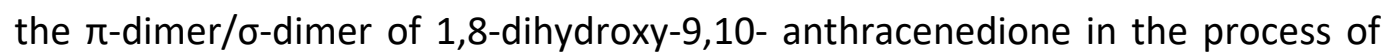
electrochemical reduction by using IR spectroelectrochemical cyclic voltabsorptometry and derivative cyclic voltabsorptometry. J Phys Chem C 117:3940-3948. https://doi.org/10.1021/jp3116947

38. Malik MA, Miecznikowski K, Marassi R, Zamponi S, Kulesza PJ, Berrettoni M (2002) Spectroelectrochemical identity of Prussian blue films in various electrolytes: comparison of time-derivative voltabsorptometric responses with conventional cyclic voltammetry. J Solid State Electrochem 1:88-93. https://doi.org/10.1007/s100080050027

39. Prado TM d., Cincotto FH, Machado SAS (2017) Spectroelectrochemical study of acetylsalicylic acid in neutral medium and its quantification in clinical and environmental samples. Electrochim Acta 233:105-112. https://doi.org/10.1016/j.electacta.2017.03.038

40. Bro R (1997) PARAFAC. Tutorial and applications. Chemom Intell Lab Syst 38:149-171. https://doi.org/10.1016/S0169-7439(97)00032-4

41. Murphy KR, Stedmon CA, Graeber D, Bro R (2013) Fluorescence spectroscopy and multi-way techniques. PARAFAC. Anal Methods 5:6557-6566. https://doi.org/10.1039/c3ay41160e

42. Bro R (2006) Review on multiway analysis in chemistry - 2000-2005. Crit Rev Anal Chem 36:279-293. https://doi.org/10.1080/10408340600969965 
43. Andersson CA, Bro R (2000) The N-way Toolbox for MATLAB. Chemom Intell Lab Syst 52:1-4. https://doi.org/10.1016/S0169-7439(00)00071-X

44. Owens JL, Marsh HA, Dryhurst G (1978) Electrochemical oxidation of uric acid and xanthine. An investigation by cyclic voltammetry, double potential step chronoamperometry and thin-layer spectroelectrochemistry. J Electroanal Chem 91:231-247. https://doi.org/10.1016/S0022-0728(78)80103-X

45. Rueda M, Aldaz A, Sanchez-Burgos F (1978) Oxidation of L-ascorbic acid on a gold electrode. Electrochim Acta 23:419-424. https://doi.org/10.1016/0013$4686(78) 87040-6$

46. Pisoschi AM, Pop A, Serban Al, Fafaneata C (2014) Electrochemical methods for ascorbic acid determination. Electrochim Acta 121:443-460. https://doi.org/10.1016/j.electacta.2013.12.127

47. Marsh HA, Dryhurst G (1979) Enzymatic and electrochemical oxidation of uric acid. J Electroanal Chem Interfacial Electrochem 95:81-90. https://doi.org/10.1016/s0022-0728(79)80221-1

48. Zhang F, Dryhurst G (1993) Oxidation chemistry of dopamine: possible insights into the age-dependent loss of dopaminergic nigrostriatal neurons. Bioorg Chem 21:392-410. https://doi.org/10.1006/bioo.1993.1033

49. Barreto WJ, Ponzoni S, Sassi P (1999) A Raman and UV-Vis study of catecholamines oxidized with Mn(III). Spectrochim Acta - Part A Mol Biomol Spectrosc 55:65-72. https://doi.org/10.1016/S1386-1425(98)00164-4

50. Bro R, Kiers HAL (2003) A new efficient method for determining the number of 
components in PARAFAC models. J Chemom 17:274-286.

https://doi.org/10.1002/cem.801 